\title{
The effect of kefir and milk intake on intestinal permeability
}

\author{
Karin Novak, Zala Jenko Pražnikar, Ana Petelin \\ University of Primorska, Faculty of Health Sciences, Polje 42, 6310 Izola, Slovenia \\ karin.novak@fvz.upr.si, zala.praznikar@fvz.upr.si, ana.petelin@fvz.upr.si
}

\begin{abstract}
We performed a longitudinal intervention study that lasted 4 months at the UP Faculty of Health Sciences in Izola. The participants were divided into two groups, each alternately consuming kefir or milk for 21 days, with two washout periods in-between. Venous blood was sampled (fasted) after each phase and the participants completed a questionnaire on gastrointestinal symptoms and a Bristol stool scale. Kefir supplementation resulted in a greater improvement of serum zonulin levels whereas significant yet similar improvement in lipid profile, serum glucose levels, inflammation markers and appetite were found in both supplementations. We did not observe significant changes in the incidence of gastrointestinal symptoms, with the exception of significantly decreased sensation of bloating after kefir intake. The average number of defecations increased after kefir intake and decreased after milk intake. The percentage of participants who defecated at a consistent time each day decreased after kefir intake and increased after milk intake. We conducted one of the first studies comparing the effects of kefir and milk on the human intestine. Our research shows that daily kefir consumption could be an effective strategy for individuals with constipation and bloating. There is still a lack of solid evidence for the use of probiotics for most medical conditions.
\end{abstract}

Keywords: kefir, milk, zonulin, intestinal permeability, inflammation

\section{Introduction}

Cow's milk and fermented dairy products, such as yogurt, buttermilk and kefir, have been a major part of our diet for many years (Kok and Hutkins, 2018) and have beneficial health effects (Bourrie et al., 2016). The intake of dairy products has been linked with reduced risk of obesity, hypertension, type 2 di- 
abetes and heart disease ( $\mathrm{Li}$ et al., 2018). Kefir is a fermented dairy product containing a large number of lactic acid bacteria and yeasts (Kim et al., 2017). The largest bacterial community in the human body is located in the gastrointestinal tract (Bischoff et al., 2014) and it's known that a diverse gut microbiota and increased intestinal permeability plays a key role in inflammation that accompanies chronic diseases and in maintaining health (Kim et al., 2017). It modulates the expression of many genes in the gut that are associated with immunity, nutrient absorption, energy metabolism, and gut barrier function (Bell et al., 2018). The human gut is surrounded by a layer of epithelial cells that forms a barrier between the environment and the host. Increased intestinal permeability is thought to be an important element in the pathogenesis of chronic inflammatory diseases, in addition to genetic and environmental factors. Zonulin is so far the only known physiological intercellular modulator of intestinal tight junctions (Sturgeon and Fasano, 2016) and it's a biomarker showing altered intestinal barrier function in many autoimmune, neurodegenerative and tumor diseases (Fasano, 2012). The specific pathophysiological role of zonulin in various diseases is not well known, but elevated zonulin levels are thought to be a key factor in the onset of the inflammatory process (Sturgeon and Fasano, 2016). Despite the prevalence of dairy products, there has been very little research done on the effects of kefir and milk intake on the intestine, especially in humans.

\section{Methods}

We performed a longitudinal intervention study, which lasted from March to June 2018 at the Faculty of Health Sciences in Izola. The study included 28 healthy individuals (13 men and 15 women) aged between 31 and 64 years, with a BMI between 25 and $30 \mathrm{~kg} / \mathrm{m}^{2}$. The exclusion criteria were: any acute or chronic illness, lactose intolerance or milk allergy, taking medication, BMI $<25 \mathrm{~kg} /$ $\mathrm{m}^{2}$ or BMI $>30 \mathrm{~kg} / \mathrm{m}^{2}$, pregnancy and lactation, significant body weight change in the last 3 months. The participants were divided into two groups, each alternately consuming kefir ( $250 \mathrm{ml}$ daily) or milk ( $250 \mathrm{ml}$ daily) for 21 days, with two washout periods in-between. During the washout periods complete abstitence from fermented dairy products was required. After each phase venous blood was sampled (fasted) and was then analyzed with Cobas c 111 analyzer (Roche Diagnostics) and with enzyme-linked immunosorbent assay (ELISA) for serum zonulin levels. After each phase participants completed a questionnaire on gastrointestinal symptoms - nausea, bloating, borborygmus, stomach pain, flatulence and heartburn. They also completed a Bristol stool scale. Participants were instructed to try to keep their diet as normal as possible throughout the study. 


\section{Results}

\section{Serum zonulin levels}

Table 1 shows the mean and standard deviation of serum zonulin values before and after kefir and milk intervention.

Table 1: Mean serum zonulin levels before and after kefir and milk intervention

\begin{tabular}{lcccc} 
& Before kefir & After kefir & Before milk & After milk \\
\hline Mean & Mean & Mean & Mean \\
\hline $\begin{array}{l}\text { Serum zonulin levels } \\
(\mathrm{ng} / \mathrm{ml})\end{array}$ & $\mathbf{1 , 0 8 9 \pm 1 , 4 2 5}$ & $0,940 \pm 0,923$ & $0,866 \pm 0,864$ & $0,837 \pm 0,913$ \\
\hline
\end{tabular}

The results of the Wilcoxon test of predicted ranks showed that both kefir intervention $(z=-0,222 ; p=0,824)$ and milk intervention $(z=-0,336 ; p=0,737)$ did not have a statistically significant effect on the change in serum zonulin levels.

Analysis of covariance (ANCOVA), with gender, age, BMI and baseline zonulin levels as covariates, showed that the change in serum zonulin levels after kefir intervention was statistically significant ( $\mathrm{p}<\mathrm{o,oo1})$, but not after milk intervention $(\mathrm{p}=\mathrm{o}, 162)$. Kefir supplementation resulted in a greater improvement of serum zonulin levels whereas significant yet similar improvement in lipid profile, serum glucose levels, inflammation markers and appetite were found in both supplementations. CRP and adiponectin were not significantly affected by neither of the two interventions (Jenko Pražnikar et al., 2020).

\section{Gastrointestinal symptoms}

Table 2 shows the mean value of the occurrence of gastrointestinal symptoms before and after kefir and milk intervention and the results of paired-samples t-test. It showed a statistically significant change only in the feeling of bloating before and after the kefir intervention $(\mathrm{p}=\mathrm{o}, \mathrm{o} 43)$, whereas the other changes in the incidence of gastrointestinal symptoms were not statistically significant.

Table 2: The occurrence of gastrointestinal symptoms before and after kefir and milk intervention and paired-samples t-test

\begin{tabular}{lcccccccc} 
& Before kefir & After kefir & $\begin{array}{c}\text { Paired-samples } \\
\text { t-test }\end{array}$ & $\begin{array}{c}\text { Before } \\
\text { milk }\end{array}$ & After milk & Paired samples t-test \\
\hline $\begin{array}{c}\text { Gastrointes- } \\
\text { tinal symp- } \\
\text { toms }\end{array}$ & Mean & Mean & $t$ & $p$ & Mean & Mean & $t$ & $p$ \\
\hline Nausea & $0,29 \pm 0,76$ & $0,29 \pm 0,71$ & 0,000 & 1,000 & $0,12 \pm 0,43$ & $0,31 \pm 0,79$ & $-1,044$ & 0,306 \\
\hline Bloating & $1,00 \pm 0,96$ & $0,70 \pm 0,87$ & 2,126 & 0,043 & $0,92 \pm 0,93$ & $1,13 \pm 1,15$ & $-0,816$ & 0,423 \\
\hline Borborygmus & $0,92 \pm 0,94$ & $0,92 \pm 0,94$ & 0,000 & 1,000 & $0,84 \pm 0,80$ & $0,60 \pm 0,96$ & 1,186 & 0,247 \\
\hline
\end{tabular}




\begin{tabular}{|c|c|c|c|c|c|c|c|c|}
\hline \multirow{2}{*}{$\begin{array}{c}\text { Gastrointes- } \\
\text { tinal symp- } \\
\text { toms* }\end{array}$} & \multirow{2}{*}{$\begin{array}{c}\text { Before kefir } \\
\text { Mean }\end{array}$} & \multirow{2}{*}{$\begin{array}{c}\text { After kefir } \\
\text { Mean }\end{array}$} & \multicolumn{2}{|c|}{$\begin{array}{c}\text { Paired-samples } \\
\text { t-test }\end{array}$} & \multirow{2}{*}{$\begin{array}{l}\text { Before } \\
\text { milk }\end{array}$} & \multirow{2}{*}{$\begin{array}{c}\text { After milk } \\
\text { Mean }\end{array}$} & \multicolumn{2}{|c|}{ Paired samples $t$-test } \\
\hline & & & $t$ & $p$ & & & $t$ & $p$ \\
\hline Stomach pain & $0,74 \pm 1,06$ & $0,52 \pm 0,89$ & 1,185 & 0,247 & $0,64 \pm 0,92$ & $0,50 \pm 0,93$ & 0,485 & 0,632 \\
\hline Flatulence & $1,50 \pm 0,91$ & $1,35 \pm 0,94$ & 0,811 & 0,425 & $1,52 \pm 0,92$ & $1,52 \pm 1,05$ & o,ooo & 1,000 \\
\hline Heartburn & $0,89 \pm 1,07$ & $0,58 \pm 0,90$ & 1,397 & 0,175 & $0,72 \pm 0,94$ & $0,56 \pm 0,87$ & 0,778 & 0,444 \\
\hline
\end{tabular}

Legend: ${ }^{*}$ Gastrointestinal symptom values are expressed on a scale from o to 5 , with 0 representing the absence of a gastrointestinal symptom and 5 representing the maximum intensity of occurrence of a gastrointestinal symptom.

An independent samples t-test to compare changes in the occurrence of gastrointestinal symptoms after intervention with kefir and milk has shown no statistically significant difference in the the altered incidence of nausea $(t$ $=-0,895 ; \mathrm{p}=0,375)$, bloating $(\mathrm{t}=-1,788 ; \mathrm{p}=0,080)$, borborygmus $(\mathrm{t}=1,051 ; \mathrm{p}=$ $0,298)$, stomach pain $(t=-0,712 ; \mathrm{p}=0,480)$, flatulence $(\mathrm{t}=-0,786 ; \mathrm{p}=0,435)$ and heartburn $(t=-0,907 ; p=0,369)$ between the groups.

\section{Stool consistency, frequency and time of defecation}

Table 3 shows stool consistency, frequency and time of defecation. Participants rated their stool consistency with a Bristol stool scale from 1 to 7 , with 1 and 2 representing harder stool, 3 and 4 stool of appropriate consistency, and 5, 6 and 7 representing softer stool. Stool consistency became softer after both kefir and milk intervention. In each stage of the study participants defecated on average from 0,5 to 3 times per day. Frequency of defecation was slightly increased after kefir intervention and decreased after milk intervention. The percentage of participants who defecated at a consistent time each day decreased after kefir intervention and increased after milk intervention.

Table 3: Average number of defecation before and after intervention with kefir and milk

\begin{tabular}{lcccc} 
& Before kefir & After kefir & Before milk & After milk \\
\hline Stool consistency & 3,6 & 3,8 & 3,8 & 3,9 \\
\hline Number of defecations & 1,3 & 1,4 & 1,4 & 1,3 \\
\hline $\begin{array}{l}\text { Defecating at a consistent } \\
\text { time (\%) }\end{array}$ & 78,3 & 70,0 & 69,9 & 77,3 \\
\hline
\end{tabular}

\section{Discussion}

Kefir intervention resulted in a greater improvement of serum zonulin levels than milk intervention. Some studies have found beneficial effects of various probiotics on the serum zonulin levels and intestinal permeability (Liu et al., 
2013; Stenman et al., 2016), while some haven't (Stadlbauer et al., 2015; Mokkala et al., 2018). CRP and adiponectin were not significantly affected by neither of the two interventions. We did not observe significant changes in the incidence of gastrointestinal symptoms, with the exception of significantly decreased sensation of bloating after kefir intervention. In most studies, consumption of kefir or other probiotics had beneficial effects on most gastrointestinal symptoms (Kato-Kataoka et al., 2016; Lee et al., 2018; Yllmaz et al., 2019). Stool consistency became softer after both kefir and milk intervention. The average number of defecations increased after kefir intervention and decreased after milk intervention. The percentage of participants who defecated at a consistent time each day decreased after kefir intervention and increased after milk intervention. Some other studies also found a beneficial effect of both kefir and milk on stool consistency or there were no differences compared to the control group (Moreira et al., 2017; Maki et al., 2018), while some studies have shown a beneficial effect of kefir or other probiotics on stool consistency, with the effect being significantly different from the control group (Lee et al., 2018; Yoon et al., 2018).

\section{Conclusions}

We conducted one of the first studies comparing the effects of kefir and milk on zonulin levels and gastrointestinal symptoms in healthy asymptomatic individuals. We have shown that daily consumption of kefir could be an effective strategy for individuals with constipation and bloating problems. There is still a lack of solid evidence for the use of probiotics for most medical conditions.

\section{References}

Bell, V., Ferrão, J., Pimentel, L., Pintado, M. and Fernandes, T. 2018. One health, fermented foods and gut microbiota. Foods, vol. 7, no. 12.

Bischoff, S. C., Barbara, G., Buurman, W., Ockhuizen, T., Schulzke, J.-D., Serino, M., ... and Wells, J. M. 2014. Intestinal permeability - a new target for disease prevention and therapy. BMC Gastroenterology, vol. 14, pp. 1-25.

Bourrie, B. C. T., Willing, B. P. and Cotter, P. D. 2016. The microbiota and health promoting characteristics of the fermented beverage kefir. Frontiers in microbiology, vol. 7, pp. 1-17.

Fasano, A. 2012. Zonulin, regulation of tight junctions, and autoimmune diseases. Annals of the New York academy of sciences, vol. 1258, pp. 25-33.

Jenko Pražnikar, Z., Kenig, S., Vardjan, T., Černelič Bizjak, M. and Petelin, A. 2020. Effects of kefir or milk supplementation on zonulin in overweight subjects. Journal of dairy science, vol. 103, no. 5, pp. 3961-3070.

Kato-Kataoka, A., Nishida, K., Takada, M., Kawai, M., Kikuchi-Hayakawa, H., Suda, K., ... and Rokutan, K. 2016. Fermented milk containing Lactobacillus casei strain Shirota preserves the diversity of the gut microbiota and relieves abdominal dysfunction in healthy medical students exposed to 
academic stress. Applied and environmental microbiology, vol. 82, no. 12, pp. 3649-3658.

Kim, D.-H., Kim, H., Jeong, D., Kang, I.-B., Chon, J.-W., Kim, H.-S., ... and Seo, K.-H. 2017. Kefir alleviates obesity and hepatic steatosis in high-fat diet-fed mice by modulation of gut microbiota and mycobiota: targeted and untargeted community analysis with correlation of biomarkers. The journal of nutritional biochemistry, vol. 44, pp. 35-43.

Kok, C. R. and Hutkins, R. 2018. Yogurt and other fermented foods as sources of health-promoting bacteria. Nutrition reviews, vol. 76, no. 1, pp. 4-15.

Lee, S.-H., Joo, N.-S., Kim, K.-M. and Kim, K.-N. 2018. The therapeutic effect of a multistrain probiotic on diarrhea-predominant irritable bowel syndrome: a pilot study. Gastroenterology research and practice, vol. 2018, pp. $1-8$.

Li, X., Yin, J., Zhu, Y., Wang, X., Hu, X., Bao, W., ... and Liu, L. 2018. Effects of whole milk supplementation on gut microbiota and cardiometabolic biomarkers in subjects with and without lactose malabsorption. Nutrients, vol. 10, no. 10, pp. 1-14.

Liu, Z.-H., Huang, M.-J., Zhang, X.-W., Wang, L., Huang, N.-Q., Peng, H., ... and Wang, J.-P. 2013. The effects of perioperative probiotic treatment on serum zonulin concentration and subsequent postoperative infectious complications after colorectal cancer surgery: a double-center and double-blind randomized clinical trial. The American journal of clinical nutrition, vol. 97, no. 1, pp. 117-126.

Maki, R., Matsukawa, M., Matsuduka, A., Hashinaga, M., Anai, H., Yamaoka, Y., ... in Fujii, C. 2018. Therapeutic effect of lyophilized, kefir-fermented milk on constipation among persons with mental and physical disabilities. Japan journal of nursing science, vol. 15, no. 3, pp. 218-225.

Mokkala, K., Pussinen, P., Houttu, N., Koivuniemi, E., Vahlberg, T. and Laitinen, K. 2018. The impact of probiotics and n-3 long-chain polyunsaturated fatty acids on intestinal permeability in pregnancy: a randomised clinical trial. Beneficial microbes, vol. 9, no. 2, pp. 199-208.

Moreira, T. R., Leonhardt, D. and Conde, S. R. 2017. Influence of drinking a probiotic fermented milk beverage containing Bifidobacterium animalis on the symptoms of constipation. Arquivos de gastroenterologia, vol. 54, no. 3, pp. 206-210.

Stadlbauer, V., Leber, B., Lemesch, S., Trajanoski, S., Bashir, M., Horvath, A., ... and Sourij, H. 2015. Lactobacillus casei Shirota supplementation does not restore gut microbiota composition and gut barrier in metabolic syndrome: a randomized pilot study. PloS one, vol. 10, no. 10, pp. 1-14.

Stenman, L. K., Lehtinen, M. J., Meland, N., Christensen, J. E., Yeung, N., Saarinen, M. T., ... and Lahtinen, S. 2016. Probiotic with or without fiber controls body fat mass, associated with serum zonulin, in overweight 
and obese adults - randomized controlled trial. EBioMedicine, vol. 13, pp. 190-200.

Sturgeon, C. and Fasano, A. 2016. Zonulin, a regulator of epithelial and endothelial barrier functions, and its involvement in chronic inflammatory diseases. Tissue barriers, vol. 4, no. 4, pp. 1-19. https://doi.org/10.108o/ 21688370.2016.1251384

Yılmaz, İ., Dolar, M. E. and Özpınar, H. 2019. Effect of administering kefir on the changes in fecal microbiota and symptoms of inflammatory bowel disease: a randomized controlled trial. The Turkish journal of gastroenterology, vol. 30 , no. 3, pp. 242-253.

Yoon, J. Y., Cha, J. M., Oh, J. K., Tan, P. L., Kim, S. H., Kwak, M. S., ... and Shin, H. P. 2018. Probiotics ameliorate stool consistency in patients with chronic constipation: a randomized, double-blind, placebo-controlled study. Digestive diseases and sciences, vol. 63, no. 10, pp. 2754-2764. 